\title{
Higher maternal age was associated with increased risks for fetal death and ectopic pregnancy
}

Nybo Andersen AM, Wohlfahrt J, Christens P, et al. Maternal age and fetal loss: population based register linkage study. BMJ 2000 Jun 24;320:1708-12.

\section{QUESTION: In pregnant women, what is the extent of risk from maternal age for fetal death (spontaneous abortion, ectopic pregnancy, or stillbirth)?}

Design

Population based study with linkage of 3 national registries for 1978 to 1992.

\section{Setting}

Denmark.

\section{Participants}

634272 women who were 12 to $\geqslant 45$ years of age and had 1221546 pregnancies between 1978 and 1992.

\section{Assessment of risk factors}

Maternal age at conception and reproductive history (parity and reproductive outcomes in the past 10 years). The calendar year was assessed as a potential confounder.

\section{Main outcome measures}

Age related risk for spontaneous abortion, ectopic pregnancy, and stillbirth. Information on reproductive outcome was obtained from the national discharge register (spontaneous abortion, hydatidiform mole, and ectopic pregnancy), the medical birth registry (live birth and stillbirth), and the national register of induced abortions. The analysis for the combined effect of maternal age and reproductive history on fetal death was limited to reproductive outcomes between 1988 and 1992.

\section{Main results}

Between 1978 and 1992, 126673 pregnancies resulted in fetal loss, 285022 in induced abortions, and 809762 in live births; the overall risk for fetal death was $13.5 \%$. The overall risk for spontaneous abortion was 10.9\%, and the risk varied according to maternal age $(8.7 \%$ for $22 \mathrm{y}$ of age to $84.1 \%$ for $\geqslant 48 \mathrm{y}$ of age). The association between maternal age and risk for spontaneous abortion was not confounded by calendar year or maternal birth cohort, and it remained when stratified by parity status and number of previous spontaneous abortions. The overall risk for ectopic pregnancy was $2.3 \%$. An association was seen between increasing maternal age and a higher risk for spontaneous abortion. The risk for ectopic pregnancy at 21 years of maternal age was $1.4 \%$, whereas the risk at $\geqslant 44$ years of age was $6.9 \%$. The overall risk for stillbirth was 4.3 per 1000 women. A J shaped relation existed between maternal age and stillbirth, but the association was not as strong as for spontaneous abortion and ectopic pregnancy.

\section{Conclusion}

Higher maternal age was associated with increased risks for spontaneous abortion, ectopic pregnancy, and stillbirth.

\section{COMMENTARY}

The study by Nybo Andersen $e t a l$ is undoubtedly generalisable. Because of its population based design, it provides the best estimates to date of the incidence of pregnancy loss and its association with maternal age and previous reproductive history.

The authors do not attempt to explain their findings. The cause of miscarriages in older women now seems to be well understood: chromosomal deletions and fatal trisomies resulting from non-disjunction during meiosis are probably the underlying biological mechanisms. ${ }^{1}$ Whether these mechanisms also explain the increased likelihood of ectopic pregnancy in older women remains to be seen. The study by Nybo Andersen et al favours the hypothetical role of the fertilised egg itself in the pathogenesis of ectopic nidation. Accumulation of other risk factors with increasing age (ie, those impairing tubal function) might also explain the association. ${ }^{2}$

The relation between stillbirth and maternal age, straightforward as it may seem, is much more difficult to comprehend. Stillbirths may result from a wide variety of underlying causes, such as vascular compromise with ensuing placental insufficiency and congenital malformations. Stillbirths represent only those cases in which obstetric intervention came too late or was deliberately withheld. Consequently, the study shows only the tip of the iceberg with respect to the true effect of maternal age on obstetric problems. If perinatal mortality had been the focus of the study (ie, including intrapartum and neonatal deaths), the prospects for older women would have been even grimmer. $^{34}$

The authors conclude that the current tendency to postpone pregnancy increases the overall incidence of fetal loss and possibly the costs of health care. Information about these findings should be part of counselling in decisions about reproduction. Apart from these messages, the paper contains reference data for all researchers in the field of human reproduction and is bound to become a touchstone study.

Willem M Ankum, MD, PhD Academic Medical Centre Amsterdam, the Netherlands

1 Volarcik K, Sheean L, Goldfarb J, et al. The meiotic competence of in-vitro matured human oocytes is influenced by donor age: evidence that folliculogenesis is compromised in the reproductively aged ovary. Hum Reprod 1998;13:15460.

2 Ankum WM, Mol BW, Van der Veen F, et al. Risk factors for ectopic pregnancy: a meta-analysis. Fertil Steril 1996;65:1093-9.

3 Forssas E, Gissler M, Sihvonen M, et al. Maternal predictors of perinatal mortality: the role of birthweight. Int J Epidemiol 1999;28:475-8.

4 Huang DY, Usher RH, Kramer MS, et al. Determinants of unexplained antepartum fetal deaths. Obstet Gynecol 2000;95:215-21. 